\title{
Placebo Analgesia From a Rubber Hand
}

Article in The journal of pain: official journal of the American Pain Society · April 2017

DOI: 10.1016/j.jpain.2017.04.004

CITATIONS

0

3 authors, including:

\section{Matthew James Coleshill}

University of Sydney

2 PUBLICATIONS 0 CITATIONS

SEE PROFILE
READS

7

All content following this page was uploaded by Matthew James Coleshill on 27 April 2017. 


\section{Accepted Manuscript}

Placebo Analgesia from a Rubber Hand

M.J. Coleshill, D.N. George, G. Mazzoni

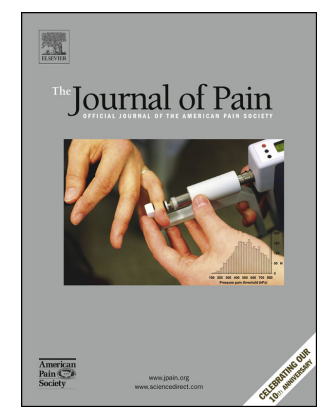

PII: S1526-5900(17)30558-8

DOI: $\quad$ 10.1016/j.jpain.2017.04.004

Reference: YJPAI 3413

To appear in: Journal of Pain

Received Date: 7 October 2016

Revised Date: 31 March 2017

Accepted Date: 10 April 2017

Please cite this article as: Coleshill MJ, George DN, Mazzoni G, Placebo Analgesia from a Rubber Hand, Journal of Pain (2017), doi: 10.1016/j.jpain.2017.04.004.

This is a PDF file of an unedited manuscript that has been accepted for publication. As a service to our customers we are providing this early version of the manuscript. The manuscript will undergo copyediting, typesetting, and review of the resulting proof before it is published in its final form. Please note that during the production process errors may be discovered which could affect the content, and all legal disclaimers that apply to the journal pertain.

(C) 2018. This manuscript version is made available under the CC-BY-NC-ND 4.0 license http:// creativecommons.org/licenses/by-nc-nd/4.0/ 
Title: Placebo Analgesia from a Rubber Hand

Authors: M. J. Coleshill ${ }^{a, b^{*}}$, D. N. George ${ }^{a, c}$ \& G. Mazzoni ${ }^{a}$

\section{Affiliations:}

aDepartment of Psychology, University of Hull, United Kingdom, HU6 7RX

${ }^{\mathrm{b}}$ School of Psychology, The University of Sydney, Sydney, New South Wales 2006, Australia

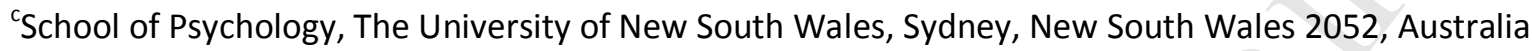

\section{Corresponding author:}

\section{Dr Matthew Coleshill}

School of Psychology

The University of Sydney

Sydney

New South Wales 2006

Australia

matthew.coleshill@sydney.edu.au

+61286274259

\section{Disclosures:}

The authors certify that there are no financial or non-financial conflicts of interest in regard to the subject matter of this research report. This work was funded by the Department of Psychology at the University of Hull with funds approved by the Departmental Research Committee. 


\section{Abstract}

Placebo analgesia, reductions in pain following administration of an inert treatment, is a welldocumented phenomenon. We report the first demonstration that placebo analgesia can be experienced when a sham analgesic is applied onto a rubber hand. The effect was obtained by exploiting the rubber hand illusion, in which ownership is felt over a rubber arm that is unattached to the body. Under conditions of both synchronous and asynchronous visuo-tactile stimulation, a thermal pain stimulus was delivered on the real arm of twenty participants and seemingly also on the rubber arm, before and after applying a sham analgesic and a control cream only to the rubber arm. During synchronous visuo-tactile stimulation, pain was experienced on the rubber arm, and the application of the sham analgesic to the rubber arm significantly decreased the severity of reported pain. This demonstrates that experience of the body can modulate expectations and the induction of placebo analgesia.

\section{Perspective}

This article presents an experiment suggesting that a placebo treatment applied to a rubber hand during the rubber hand illusion can produce placebo analgesia. This finding indicates that embodiment may influence the placebo effect, a previously unexamined factor in the treatment process with potential applications to treatment administration.

Keywords: Placebo Effect, Placebo Analgesia, Rubber Hand Illusion, Body Ownership, Embodiment 


\section{Introduction}

The mechanisms through which our beliefs alter our perception and physiological processes remains an intriguing area of psychological research, with the placebo effect being a prime example of this phenomenon. The placebo effect is the portion of therapeutic improvement attributable to psychobiological components following the receipt of an inert treatment, distinct from factors such as spontaneous remission and response bias. ${ }^{6}$ One of the best understood placebo effects is placebo analgesia, which is a robust phenomenon during both active pharmacological ${ }^{72}$ and inert treatments. ${ }^{44}$ Placebo analgesia is mediated by conscious expectations, influenced by forms of learning such as verbal cues, classical conditioning, and social observation. ${ }^{14}{ }^{15}{ }^{48} \mathrm{~A}$ recent meta-analysis ${ }^{2}$ indicates that these conscious expectations mediate placebo analgesia through prefrontal regions, ${ }^{41}{ }^{52}$ leading to activation of the descending pain modulatory system. ${ }^{7} 18$ This network then employs the endogenous opioid system and $\mu$-opioid neurotransmission ${ }^{5}$ decreasing activity in pain-processing regions ${ }^{52}$ as well as modulating nociceptive processing at the spinal level, ${ }^{19}{ }^{40}$ inducing analgesia.

To date, extensive research has examined how expectations influence both the occurrence and degree of placebo analgesia. ${ }^{48}$ Such studies have focused on manipulating the treatment itself, either through its characteristics, ${ }^{9}{ }^{16}$ or through pharmacological ${ }^{16}$ or non-pharmacological conditioning procedures. ${ }^{44}$ No research, however, has examined how experience of the body, as opposed to perception of the treatment itself or its efficacy, influences expectations and placebo analgesia. A potential avenue for such investigations is the experimental manipulation of experience of ownership felt over the body, obtained by controlling the information inputted into multisensory processing systems. An ideal example of this is the rubber hand illusion (RHI), in which an artificial arm not connected to the body is subjectively experienced as part of the body through the integration of 
temporally and spatially congruent tactile and visual information. ${ }^{49}$ The $\mathrm{RHI}$ is one of the few reliable scientific paradigms to investigate body ownership, and has been extensively studied both behaviourally and neurologically. ${ }^{50}$ Those subject to this phenomenon report touch and pain ${ }^{11}$ occurring on the rubber hand alongside a sense of ownership. ${ }^{89} 50$ Further, threatening the rubber hand during the illusion modulates anxiety-related activity in the anterior cingulate cortex, ${ }^{17}$ suggesting that interactions with the artificial limb may influence affective responses, altering pain-related neural activity. Indeed, recent research also indicates that the application of acupuncture to the limb during experience of the illusion produces subjective sensations associated with this treatment which were correlated with neural activity in the interoceptive system. ${ }^{12} 13$

Given that the limb is experienced as part of the body, that patterns of neural activity appear to reflect some integration with the pain matrix, and that other somatosensory networks respond to interactions with the limb, it appears possible that a treatment applied to the artificial limb itself could modulate expectations, and thus, the experience of pain. The present study aimed to test this hypothesis using a procedure in which the RHI was induced during a conditioned placebo paradigm, wherein the placebo and control treatments were applied to the artificial limb itself. This procedure was completed under conditions of both synchronous and asynchronous visuo-tactile stimulation.

\section{Methods}

\section{Participants}

Power analysis, using an alpha level of $5 \%$ and power of $95 \%$, was conducted on the pooled effect size of studies investigating placebo analgesia between 2002 and $2007\left(d=0.81^{51}\right)$. This estimated that a sample size of 20 would demonstrate a similar effect of a placebo treatment. Based on the literature regarding the $\mathrm{RHI}^{8}{ }^{8}$ this sample size was also sufficient to observe an effect of the illusion. Twenty righthanded undergraduate students (13 female) from the University of Hull (mean age $21.25, S D=6.06$, 
range $=18-43)$ participated in the experiment for course credit. Participants were reimbursed $f 16$ for their participation. Exclusion criteria for the experiment, listed on the advertisement and confirmed upon arrival, were the current use of any prescribed or unprescribed analgesics, any previous or existing pain condition, as well as the right-hand being the non-dominant hand. Ethical approval was provided by the University of Hull departmental ethical committee and conformed to British Psychological Society guidelines.

\section{Design}

The experiment used a 2 (synchronous tactile stimulation, asynchronous tactile stimulation) $\times 2$ (placebo treatment, control treatment) $\times 3$ (pre-treatment, conditioning, post-treatment) repeated measures design. Participants experienced two sessions of placebo and control treatments differing in the synchronicity of tactile stimulation, each involving three phases of nociceptive stimulation.

\section{Rubber Hand IIlusion}

A partition placed on a table to the right of the participants' midline blocked the view of the real arm, 15 $\mathrm{cm}$ away on the left of the partition was placed an artificial limb. Two rubber hands were used, one female (42 cm in length) and one male (49 cm in length) (right-handed PVC prosthetic arm covers filled with foam beads [RSL Steeper, Leeds, United Kingdom]).

In the synchronous condition the participant's and rubber hand's middle fingers were stimulated in a synchronous fashion using a repeating cycle of a stroke (distally from the dorsal proximal interphalangeal to the eponychium), a tap (directed toward the dorsal proximal interphalangeal), and a lift (raising the finger by $30^{\circ}$ before letting it drop back to the table). Each cycle was completed at a rate of $0.3 \mathrm{~Hz}$. In the asynchronous condition this stimulation was performed in an asynchronous fashion.

Based on the results of a pilot study, an adapted version of the Botvinick and Cohen questionnaire ${ }^{8}$ tailored to specifically address subjective sense of ownership and displacement of tactile 
and nociceptive sensation onto the artificial limb, was used to measure the RHI (Table 1). Participants were prompted to first respond 'yes' or 'no' to each question. Only after 'yes' responses they were asked to rate how strong the sensation was on a 1-5 numerical rating scale, with ' 1 ' being labelled 'very mild' and ' 5 ' being labelled 'very strong'. A 'no' response was scored as a ' 0 ' on the rating scale. This choice was added in order to ensure that an absence of the illusion was consistently rated by participants in line with some established measures of anomalous experiences. ${ }^{4}$

Proprioceptive drift was recorded using a task in which participants closed their eyes and estimated the location of their right arm beneath the table using their left hand both before and after the induction of the illusion. Under the table a $100 \mathrm{~cm}$ tape measure was attached, using which the experimenter recorded where the participant estimated their arm to be. For each participant, the baseline estimate was subtracted from the estimate made after visuo-tactile stimulation in each phase in order to measure the perceived drift in felt arm position brought about by the RHI.

\section{Thermal Stimuli}

A contact thermode (Pathway CHEPS, Medoc, Ramat Yishai, Israel) was used to accurately deliver thermal nociceptive stimuli. A second mock thermode was attached to the rubber hand, and had a builtin visual stimulus (a red LED light) which could be manually activated by the experimenter. All stimuli in the experiment used the 'Ramp and Hold' setting, increasing in temperature at a rate of $10^{\circ} \mathrm{C}$ per second from a baseline of $35^{\circ} \mathrm{C}$, before maintaining at the destination temperature for the remainder of the 5 second duration.

Participants' perception of pain was measured using two rating scales, one assessing pain intensity (sensory dimension of pain - 0 corresponded to 'No Pain'; '100' to 'Intolerable Pain') and the other pain unpleasantness (affective dimension of pain - 0 was 'Not Unpleasant At All' and 100 'Most 
Unpleasant Imaginable'). Scales were printed and placed on the table in front of the participant and remained visible throughout the experiment.

Each session started with a calibration procedure ${ }^{44}$ in order to tailor the strength of the pain stimuli to each individual. Four thermal stimuli $\left(44^{\circ} \mathrm{C}, 45^{\circ} \mathrm{C}, 47^{\circ} \mathrm{C}, \& 49^{\circ} \mathrm{C}\right)$ were each applied twice in a randomised order to the selected location, which depended on the counterbalancing order for that individual. Participants rated the pain intensity of each thermal stimulus using the aforementioned scale. Using these ratings, the temperature deemed to represent the high (60 on the numerical scale) or low (30 on the numerical scale) intensity stimulus for each participant was calculated using a regression analysis.

\section{Placebo Treatment}

Small white containers stored the inert placebo treatment and control cream. The placebo treatment was a mixture of E45 moisturising cream and alcohol sanitiser (to give the placebo treatment a chemical odour), while the control cream did not contain the alcohol sanitiser. The containers' labelling ("Hull York Medical School - Topical Anaesthetic Lidocaine - Authorized for Research Purposes Only" on the placebo treatment and "Aqueous Cream" on the control treatment) was consistent with previous research on the placebo effect. ${ }^{30} 44$ The placebo treatment was applied using surgical gloves. In order to improve the psychosocial context, the experimenter also wore a white lab coat throughout the procedure, and the study was conducted in a room labelled "Behavioural Medicine Laboratory". This pretext, environment, and means of treatment administration reliably induced a placebo effect with conditioning in pilot testing.

\section{Procedure}

The experiment was advertised as a study examining the effect of analgesics during the RHI. Participants were told that they were to rate their experience of pain in a number of pain trials during two different 
conditions of the RHI, and to complete a number of questionnaires regarding their experience of the illusion. They were also informed that after a number of trials the analgesic treatment and the control treatment would be applied to the artificial limb itself. To clarify this, participants were informed of the preliminary nature of the study, told that it was not clear what effect this analgesic could have during this procedure, and that while this was an unusual method they should continue to accurately report their genuine pain experience throughout the experiment. Participants then provided informed consent to take part in the study.

The experiment was conducted in two sessions, separated by approximately two weeks in order to reduce any effects of habituation to pain. In each session, participants were seated at a table, with both hands flat on the table in front of them. On the table was the experimental set-up for the RHI described above. At the beginning of the experiment, two sites of the real right arm (and corresponding sites on the rubber hand) were identified. These sites were medially located on the dorsal surface of the right forearm, with site A being distally located and site B in a proximal position (see Figure 1). These two locations would be used as stimulation sites where the placebo and control treatments would later be applied, with thermal stimulation alternating between the two sites. Both the thermode on the real arm and the mock thermode on the artificial limb were moved in unison so that they were always located on the corresponding site between the two limbs. The two sessions varied in regard to the synchronicity of visuo-tactile stimulation, while factors such as the initial site of thermal stimulation and the location of the placebo treatment on the rubber arm were maintained between the two sessions (i.e. a participant for whom site A was both the first site of stimulation and the location of the placebo treatment during synchronous stimulation would also undergo the same during asynchronous stimulation). The order of sessions (synchronous, asynchronous), the initial site of stimulation, and the location of the placebo treatment were all counterbalanced between participants. 
Each session consisted of 14 blocks of thermal stimulation, split into three phases, preconditioning (baseline), conditioning, and post-conditioning. Each block of stimuli contained four individual thermal stimuli (see Table 2), and alternated between the two sites on the participant's right forearm. A baseline of ' 60 ' perceived pain intensity was used.

Visuo-tactile stimulation was applied for 45 seconds before starting the thermal stimulation, and was maintained throughout each block. Measures of the RHI (questionnaires and proprioceptive drift) were taken throughout the experiment following the $3^{\text {rd }}, 4^{\text {th }}, 7^{\text {th }}, 8^{\text {th }}, 13^{\text {th }}$ and $14^{\text {th }}$ blocks. During each thermal stimulus, the red LED on the mock thermode placed on the rubber arm was manually operated by the experimenter, with the light signalling the onset and duration of each stimulus. During each of these blocks participants were instructed to focus their attention on the artificial limb, specifically where the tactile stimulation was occurring.

\section{Pre-Conditioning Phase}

During the pre-conditioning phase, the thermode and mock thermode were attached to the participant's arm and the artificial limb. Site was similarly counterbalanced on both real and rubber arm. After applying visuo-tactile stimulation, a first block of high intensity (' 60 ' perceived intensity) thermal stimuli was administered. The same procedure was repeated until two blocks of stimulation had been applied on each site. Participants verbally rated the perceived pain intensity and unpleasantness of each individual stimulus. After the $3^{\text {rd }}$ and $4^{\text {th }}$ blocks of stimulation, participants completed the RHI questionnaire and performed the measure of proprioceptive drift.

\section{Conditioning Phase}

Following completion of the pre-conditioning phase two creams were presented. Participants were told that one was a powerful analgesic called lidocaine, whereas the other was a simple moisturising cream. The sham analgesic was applied with latex gloves to the treatment site of the rubber limb. Location of 
sham analgesic and control creams on the artificial limb was counterbalanced between participants. After applying the placebo or the moisturiser, the thermode and the mock thermode were then reattached on the participant's arm and the rubber limb, with half of the participants starting with the treatment site and half with the control site. The $\mathrm{RHI}$ was then induced and the blocks of thermal stimulation repeated as during the pre-treatment phase, with the exception that stimulation at the sham analgesic site was surreptitiously applied at a low ' 30 ' perceived pain intensity. This was to induce a strong expectancy that the sham analgesic treatment would decrease perceived pain. ${ }^{34}$ This method was repeated for a total of six blocks of stimulation. Measures of the RHI were obtained following the $7^{\text {th }}$ and $8^{\text {th }}$ blocks of stimulation. Two further blocks of conditioning stimulation were then conducted after this in order to ensure the transition from the conditioning to post-conditioning phase was as seamless as possible. This was done in order to reduce detection of change in the experimental method.

\section{Post-Conditioning Phase}

This phase immediately followed the conditioning phase, with the procedure and duration being identical to the pre-treatment phase. The blocks of thermal stimulation at the site that previously received the low intensity stimulation in the conditioning phase were surreptitiously increased to the same high intensity stimulation level as that of the control site (i.e. '60' perceived pain intensity). A total of four blocks of thermal stimulation were applied in an alternating pattern between stimulation sites (see Figure 2). Measures of the RHI were collected following the completion of the post-treatment phase after the $13^{\text {th }}$ and $14^{\text {th }}$ blocks of stimuli.

\section{Statistical Analyses}

Data were collapsed across stimulation site $A$ and $B$ for the purposes of data analysis. Baseline comparisons of pain intensity and unpleasantness across conditions of synchronicity and treatment were conducted using one-way repeated measures ANOVAs. Data from all outcomes were analysed 
using 2 (synchronous, asynchronous) $\times 2$ (placebo, control) $\times 2$ (pre-treatment, post-treatment) repeated measures ANOVAs. Post-hoc analysis of these outcomes were performed using both simple effects analysis and post-hoc t-tests adjusted for multiple comparisons using the Bonferroni correction. Finally, correlational analyses were performed using Pearson bivariate correlations (two-tailed).

\section{Results}

Baseline Assessment

Baseline comparisons revealed no significant differences in pain intensity: $F(3,57)=1.146, p=.339$, or unpleasantness: $F(3,57)=1.324, p=.275$, between treatment sites or conditions of synchronicity.

\section{Rubber Hand Illusion}

All three subscales of the questionnaire (ownership, tactile embodiment, and nociceptive embodiment) were higher during synchronous as compared to asynchronous stimulation (Table 3). The range of data was ' 5 ' across all three subscales during synchronous stimulation and varied between ' 4 ' and ' 5 ' during asynchronous stimulation. A reliability analysis performed on the $\mathrm{RHI}$ questionnaire revealed Cronbach's alphas from .94 to $.96($ mean $=.95)$ during synchronous stimulation, and from .89 to .95 (mean $=.93)$ during asynchronous stimulation. A bivariate correlation analysis revealed that the three subscales were significantly correlated with each other $(p<.01$ or $p<.05)$ across all phases, at both treatment sites, and under both conditions of stimulation synchronicity (from $r=.67$ to $r=.95$ [mean $r=.81$ ] during synchronous, and from $r=.56$ to $r=.96$ [mean $r=.78$ ] during asynchronous stimulation). Experience of the RHI was considered as a rating of greater than ' 1 ' and was assessed by collapsing each subscale across both treatment sites and all three phases of the experiment. During synchronous visuo-tactile stimulation $75 \%$ of participants reported experiencing ownership over the 
rubber hand and a sense of tactile embodiment, whereas $55 \%$ reported the embodiment of pain onto the artificial limb (see Supplementary Materials for analyses with non-responders excluded).

Four three-way $2 \times 2 \times 2$ repeated measures ANOVAs were conducted on the measures of the $\mathrm{RHI}$, using synchronicity (synchronous, asynchronous), treatment (placebo, control), and phase (pretreatment, post-treatment) as within-subjects factors. The first of these examined average responses to question one, which assessed experience of ownership over the rubber hand, finding a significant main effect of synchronicity: $F(1,19)=10.074, p=.005, \eta_{p}^{2}=.346, d=0.55,95 \% \mathrm{Cl}$ for synchronous [1.59, $3.26]$ and asynchronous conditions $[0.80,2.22]$, indicating that synchronous visuo-tactile stimulation lead a greater sense of ownership over the rubber hand compared to asynchronous visuo-tactile stimulation. A significant interaction was also observed between synchronicity and treatment: $F(1,19)=$ 5.942, $p=.025, \eta_{p}^{2}=.238$, in which there appears to be a greater disparity in ownership between the synchronous and asynchronous conditions at the control site in comparison to the placebo site. All other effects and interactions failed to reach significance (largest $F(1,19)=1.355, p=.259)$.

Four post-hoc t-tests were performed to examine the interaction between synchronicity and treatment on subjective sense of ownership over the rubber hand (after a Bonferroni correction, alpha value of 0.0125 ). These analyses found that ownership was significantly greater during synchronous stimulation at both the placebo site: $t(19)=2.97, p=.008$ and the control site $: t(19)=3.58, p=.002$, compared to during asynchronous stimulation. As this difference was larger at the control site, however, it appears that a greater disparity in subjective ownership at the control site in comparison to the placebo site underlies this interaction.

Analysis of the subscale of tactile embodiment, which comprised of the averaged response to questions four and five, found no significant main effects or interactions (largest $F(1,19)=2.879, p=$ .106). The subscale of pain embodiment which comprised of the averaged response of questions two 
and three was then analysed, finding a significant main effect of synchronicity: $F(1,19)=8.836, p=.008$, $\eta_{\mathrm{p}}{ }^{2}=.317, d=0.52,95 \% \mathrm{Cl}$ for synchronous $[0.95,2.63]$ and asynchronous conditions [0.36, 1.59], suggesting that the embodiment of pain was greater during synchronous visuo-tactile stimulation compared to asynchronous stimulation. All other effects and interactions failed to reach significance (largest $F(1,19)=2.999, p=.100)$. A final ANOVA examined the behavioural measure of proprioceptive drift, finding no significant main effects or interactions (largest $F(1,19)=2.013, p=.172$ ).

\section{Pain Intensity}

Visual inspection of the data suggests that pain intensity decreased at the placebo site and increased at the control site when comparing pre and post-conditioning treatment, but only during the synchronous stimulation. A notable disparity in baseline ratings between treatment sites during asynchronous stimulation was also observed (Table 4).

The 2 (synchronous, asynchronous) $\times 2$ (placebo, control) $\times 2$ (pre-treatment, post-treatment phase) repeated measures ANOVA on average pain intensity ratings revealed a main effect of treatment: $F(1,19)=5.87, p=.026, \eta_{p}^{2}=.236$, qualified, more importantly, by a significant interaction between treatment and phase: $F(1,19)=5.28, p=.033, \eta_{p}^{2}=.237$, and a significant three-way interaction: $F(1$, $19)=14.02, p=.001, \eta_{p}{ }^{2}=.425$. All other main effects and interactions failed to reach significance $(F s<$ 1).

A simple effects analysis conducted in order to examine the three-way interaction revealed a significant effect of treatment during synchronous stimulation in the post-treatment phase: $F(1,76)=$ $16.11, \mathrm{p}<.000$, indicating that the placebo treatment decreased pain intensity but only during synchronous stimulation. It also revealed a significant effect of phase at the placebo site during synchronous stimulation: $F(1,76)=4.30, p=.042$, indicating that pain ratings decreased only at the 
placebo treatment site following receipt of the treatment, and only during synchronous stimulation. All other effects failed to reach significance (largest $F(1,76)=3.29, \mathrm{p}=.074)$.

Based on the results of the simple effects analysis, two post-hoc t-tests performed on the threeway interaction (after a Bonferroni correction, alpha value of 0.025), finding a significant difference in pain intensity ratings between the placebo and control sites in the post-treatment phase during synchronous stimulation: $t(19)=3.36, p=.002, d=0.75$. The difference in pain intensity between the pre and post-treatment phases at the placebo site during synchronous stimulation failed to reach significance: $t(19)=1.96, p=.066$. These results indicate that the decrease in pain intensity occurred only in the post-treatment phase, was specific to the placebo treated location, and was significantly stronger during synchronous than asynchronous stimulation (Figure 3).

\section{Pain Unpleasantness}

Data on pain unpleasantness were similar to data on pain intensity in that during synchronous stimulation unpleasantness decreased at the placebo site and increased at the control site, whereas during asynchronous stimulation ratings either increased or were stable. Further, a substantial disparity in baseline ratings between the two sites was observed during asynchronous stimulation (Table 5).

The $2 \times 2 \times 2$ ANOVA on average pain unpleasantness ratings also found a significant main effect of treatment: $F(1,19)=6.95, p=.016, \eta_{p}^{2}=.268$, as well as an interaction between phase and treatment, $F(1,19)=4.47, p=.048, \eta_{p}^{2}=.190$. More importantly, the three-way interaction between treatment, synchronicity and phase was significant: $F(1,19)=6.66, p=.018, \eta_{p}^{2}=.260$. The other main effects and interactions failed to reach significance $(F s<1)$.

A simple effects analysis conducted in order to examine the three-way interaction revealed a significant effect of treatment during synchronous stimulation in the post-treatment phase: $F(1,76)=$ $12.30, p=.001$, a significant effect of treatment during asynchronous stimulation in the pre-treatment 
phase: $F(1,76)=4.11, p=.046$, as well as a significant effect of phase at the control site during synchronous stimulation: $F(1,76)=5.99, p=.017$. All other effects failed to reach significance (largest $F(1,76)=3.01, p=.087)$.

Based on the results of the simple effects analysis, three post-hoc t-tests performed on the three-way interaction (after a Bonferroni correction, alpha value of 0.016), finding a significant difference in pain unpleasantness ratings between the placebo and control sites in the post-treatment phase during synchronous stimulation: $t(19)=3.16, p=.005, d=0.58$. A significant increase in unpleasantness ratings was also found between the pre and post-treatment phases at the control site during synchronous stimulation: $t(19)=3.01, p=.007, d=0.44$. The difference between the two treatment sites in the pre-treatment phase during asynchronous stimulation failed to reach significance: $t(19)=-2.19, p=.041$. This suggests that the disparity in baseline scores did not contribute significantly to the three-way interaction. These findings indicate that only during synchronous stimulation did the application of the placebo treatment lead to changes in unpleasantness ratings between the two sites, which was accompanied by significant sensitisation through the course of the experiment at the control site (Figure 4).

\section{Correlational Analysis}

In order to examine the relationship between experience of the $\mathrm{RHI}$ and the induction of placebo analgesia through the rubber arm, a correlational analysis was performed on results from the placebo treatment site during synchronous visuo-tactile stimulation. Measures of pain intensity and unpleasantness, specifically ratings during the post-treatment phase and the difference between the pre and post-treatment phases, were correlated with the three subscales of the RHI during both the pre and post-treatment phases (Table 6). Besides expected correlations within measures of pain and the RHI, this analysis revealed significant negative correlations between the difference in pain intensity from the 
pre to the post-treatment phase with pain embodiment during the post-treatment phase $(r=-.483, p=$ .031), as well as the difference in pain unpleasantness between the pre and post-treatment phases with pain embodiment during the pre-treatment phase $(r=-.467, p=.038)$. These correlations indicate that a larger reduction in pain intensity and unpleasantness following receipt of the treatment was associated with greater reporting of pain as occurring on the rubber hand during either the post or pre-treatment phases respectively.

\section{Discussion}

This is the first demonstration that placebo analgesia can be induced through the application of a treatment to an artificial limb that is ostensibly unattached to the body. Previous research on the RHI demonstrated that pain can be experienced as occurring on the rubber hand through synchronous nociceptive input, ${ }^{11}$ and that threatening the artificial limb produces patterns of neural activity similar to threatening the actual arm. ${ }^{17}$ Here we demonstrate that a reduction in pain attributable to the placebo effect is experienced when a placebo treatment is applied to the rubber arm. Crucially, and in line with

research on the $\mathrm{RHI}^{8}{ }^{80}$ this placebo effect only occurs when the $\mathrm{RHI}$ is induced, that is, during synchronous stimulation. As this effect is specific to the placebo treatment site during synchronous stimulation, it is unlikely to be a product of the visual cue on the mock thermode, attention, or the RHI itself, as such explanations would also predict a reduction in pain at the control site. As such, this finding also replicates previous research indicating that the $\mathrm{RHI}$ has no analgesic effect. ${ }^{31}$ Further, conditioned pain modulation cannot account for the results, as such an explanation would also expect a reduction in pain during asynchronous stimulation.

These findings are not only novel, but also build upon recent research on the RHI indicating that the experience of embodiment over the rubber hand may interact with the top-down modulation of sensory experience. In particular, research examining the application of acupuncture to the rubber hand 
found that it may produce subjective sensations associated with this form of treatment, as demonstrated by correlations between these measures and activity in a variety of regions associated with the RHI. ${ }^{12}{ }^{13}$ These studies, however, only assessed subjective sensory phenomena associated with acupuncture, as opposed to its actual therapeutic efficacy. As such, the present study represents a significant advancement, as it suggests that multisensory processing, and the resulting sense of embodiment, may influence expectations regarding the efficacy of a treatment, in turn reducing symptoms through the placebo effect. In other words, when a topical treatment is applied to the artificial limb during experience of the illusion, there are increased expectations that it may influence pain perception, relative to when the illusion is absent. These expectations, bolstered by the conditioning procedure, then lead to placebo analgesia through the descending pain modulatory system.

Besides a reduction in pain only being observed during synchronous visuo-tactile stimulation, further evidence for this account can be seen in the correlations between the experience of pain as occurring on the rubber hand and changes in pain intensity and unpleasantness following receipt of the treatment. This suggests that the displacement of pain was particularly relevant to expecting a treatment applied in this manner to alter pain experience. While it is somewhat surprising that this sensation, as opposed to the sense of ownership over the rubber hand, modulated placebo analgesia, it nonetheless provides additional evidence that the illusory experience of the RHI was integral to the observed effect. To date, little is known as to how perceptual displacement of pain in the RHI relates to both ownership and the displacement of touch. Models of the RHI indicate that the referral of touch to the rubber hand based on the concurrent visual and tactile information is a prerequisite for ownership, ${ }^{50}$ though the related functionality of pain referral is unknown. One possibility is a parallel recalibration alongside touch, which raises the question of whether the displacement of pain may be induced in isolation from tactile input, as well as whether this is sufficient to induce ownership. Examining such 
questions may elucidate the displacement of pain and how it contributed to the induction of placebo analgesia in this study.

One interesting notion regarding these findings is the extent to which embodiment is a necessary prerequisite for placebo analgesia. While no research has directly addressed this possibility, there is a growing body of evidence indicating that chronic pain conditions are associated with distorted body representation. ${ }^{37}$ In particular, such distortions have been observed in complex regional pain syndrome (CRPS) ${ }^{25} 3538$ and chronic back pain. ${ }^{1036}$ If embodiment is necessary for placebo analgesia then it would be expected that such conditions would display impaired placebo effects when the treatment is applied to the afflicted region. Previous research, however, has observed considerable pain relief following receipt of a placebo treatment in both $\mathrm{CRPS}^{43}$ and chronic back pain. ${ }^{22}$ Nonetheless, it should be noted that these studies did not directly measure bodily distortions, nor did they compare the degree of pain relief with a relevant control sample, and so an association cannot be entirely ruled out.

The analgesic effect observed in this study also bears some similarities to visually induced analgesia, in which sight of the body itself can reduce pain perception. ${ }^{27}{ }^{31}$ In this phenomena, information about the body, typically visual input regarding the body part in pain, has an inherent analgesic effect. This response is driven by bottom-up processing, wherein visual information about the body, processed in parietal and occipital regions related to the representation of body, instigates the descending modulation of pain without higher-order frontal regions. ${ }^{28}$ Similar effects have also been observed using the RHI in virtual environments, ${ }^{32}{ }^{39}$ and may provide an explanation, through factors such as colocation with the body, as to inconsistencies in the literature regarding whether the RHI itself has an analgesic effect. ${ }^{23}{ }^{33}$ In contrast, the specificity of analgesia to the treatment site during synchronous visuo-tactile stimulation in the present study suggests that top-down expectations of pain relief following receipt of a treatment were modulated by whether pain was referred to the location of the treatment. The neural mechanisms underpinning this likely involve activation of the descending 
pain modulatory system through lateral prefrontal regions, ${ }^{2}$ precluded by a recalibration of felt pain through multisensory processing regions in the premotor and parietal cortices. ${ }^{50}$ In this manner, the two phenomena represent contrasting methods by which information about the body may instigate the descending modulation of pain.

As this is the first study to demonstrate an interaction between the placebo effect and embodiment, subsequent studies should also examine the boundaries of this effect, and map the extent to which similarity ${ }^{20}$ or distance ${ }^{26}$ between the rubber limb and the body, factors which modulate the RHI, may alter the degree of placebo analgesia. Future studies should also examine the role of visual information in shaping this effect, and whether placebo analgesia can be induced using the non-visual variant of the $\mathrm{RHI}^{29}$

The finding that embodiment of pain may influence treatment expectations paves the way for further, more elaborate investigations of how experience of the body may interact with the placebo effect. There is a growing field of research examining embodiment in immersive virtual reality. ${ }^{247}$ Such techniques allow for the precise control of the presented environments and pose an interesting avenue for investigating treatment expectations and embodiment. Intriguingly, recent research has found that virtual reality techniques, such as visual feedback regarding movement, may alter the experience of pain. ${ }^{21}$ The findings from the present study suggest that the application of a treatment within such a virtual environment, alongside the appropriate conditioning procedures, may have the potential to induce placebo analgesia.

The results of this study are also consistent with previous research on the placebo effect, which indicate that the manipulation of information regarding the placebo treatment, such as its characteristics ${ }^{916}$ or the perceived likelihood of receiving an active treatment, ${ }^{45}$ alters understanding of the treatment and thus affects expectations. These expectations then modulate the placebo effect. The 
present study, however, represents an intriguing demonstration of the influence of expectancy on the placebo effect, as it is the subjective experience of what is part of the body that is manipulated, rather than the characteristics of the treatment itself or the perceived likelihood of receipt. Our findings show that manipulations of bodily experience may result in placebo analgesia when the location of the treatment is moved outside of the body itself. This is a powerful demonstration of the influence of expectations on the induction of placebo analgesia, as well as a unique example of the factors that may influence expectations.

Non-pharmacological conditioning procedures have also been consistently demonstrated to be a powerful means of manipulating learning in regard to placebo analgesia. ${ }^{48}$ It may be that the conditioning procedure is essential in the present paradigm to induce the expectancy that the treatment is effective. Future studies may wish to address this question by attempting to replicate the current findings using an experimental paradigm in which the conditioning procedure is removed.

While the present study examined how treatments applied to the artificial limb may influence experimentally induced pain, an intriguing question is whether this procedure may also have clinical applications. In many ways, the present procedure represents a method of administering an intervention to a patient without direct contact with the body, albeit only in regard to inducing a placebo effect. While this is contingent on conditioning not being a necessary part of the procedure, as discussed above, this method of clinical intervention may be particularly applicable if feasible in conjunction with the virtual reality techniques discussed previously. Such a procedure may be able to instigate a placebo effect with no bodily interaction whatsoever, that is, without the need for synchronous visuo-tactile stimulation. This may be especially pertinent to conditions where interacting with the body can cause discomfort or pain, such as fibromyalgia or CPRS. Further, if successful, such a procedure may have application to diseases and disorders outside of pain which are responsive to the placebo effect. Previous research also indicates that even placebos are capable of producing unpleasant 
side effects in patients. ${ }^{36}$ As the present paradigm entails no interaction between the treatment and the body, it may have the potential to circumnavigate such side effects. Future research should aim to examine whether this is the case by comparing the rate of side effects between a placebo treatment applied to the body and one applied to an artificial limb.

Strangely, no difference was found in subjective ratings of tactile embodiment between conditions of visuo-tactile synchronicity, although a difference was noted when non-responders to the RHI were excluded. This finding is difficult to explain given the consistent replicability of this finding both within the literature in general, as well as the significant differences found within the other subjective measures. While this result may simply be explained as an anomaly within the data, it could also be a product of the questionnaire. While the changes to the questionnaire proved to be reliable during pilot testing, removal of the range of responses in regard to an absence of the experience may have reduced the overall variance and contributed to this outcome. Further, the study also failed to find any effect of visuo-tactile synchronicity on the behavioural measure of proprioceptive drift, a finding in contrast to previous studies. ${ }^{85}$ Akin to tactile embodiment, however, a difference in this measure was observed when non-responders were excluded. A potential explanation for this is that the method used in the present experiment required participants to estimate the location of their right arm using their left hand, this movement itself may have adversely affected the strength of the illusion and reduced the degree of proprioceptive drift towards the rubber hand.

While the study observed an effect of a treatment applied in this manner without excluding participants who did not respond to the RHI, it remains that the sample size itself is still small. To these ends, the results presented here represent a proof of principle regarding this experimental paradigm which should be replicated in larger cohorts. Indeed, a larger sample size may yield further correlational findings between measures of the $\mathrm{RHI}$ and placebo analgesia. Further, although the procedure was designed to avoid clear demand characteristics, another research avenue should aim to replicate the 
present findings and incorporate a surreptitious naloxone manipulation ${ }^{5}$ in order to demonstrate that the effect is mediated by neurobiological mechanisms. The present experiment also did not employ any measures of expectancy regarding the degree of pain relief. Such a measure was not used in order to avoid any association with placebo research in the participants. Nonetheless, recording expectations may have yielded interesting findings regarding the extent to which participants actually expected pain relief following the application of a placebo treatment to the artificial limb, as opposed to their own arm.

In conclusion, the present study outlines an intriguing method of inducing a placebo effect, in which a sham topical anaesthetic is applied to a rubber arm unattached to the body, but is experienced as so through an illusion of bodily ownership. Such a finding demonstrates how experience of the body may alter treatment outcomes - a previously unexamined aspect of the treatment process with potential implications for treatment administration.

\section{Acknowledgements:}

The authors would like to extend their appreciation to Dr Katja Wiech for her advice in preparing the manuscript. 


\section{References}

1. Amanzio M, \& Benedetti F: Neuropharmacological dissection of placebo analgesia: expectationactivated opioid systems versus conditioning-activated specific subsystems. The Journal of Neuroscience, 19, 484-494, 1999.

2. Amanzio M, Benedetti F, Porro C A, Palermo S, \& Cauda F: Activation likelihood estimation metaanalysis of brain correlates of placebo analgesia in human experimental pain. Human Brain Mapping, 34, 738-752, 2013.

3. Amanzio M, Corazzini L L, Vase L, \& Benedetti F: A systematic review of adverse events in placebo groups of anti-migraine clinical trials. Pain, 146, 261-269, 2009.

4. Bell V, Halligan P W, \& Ellis H D: The Cardiff anomalous perceptions scale (CAPS): A new validated measure of anomalous perceptual experience. Schizophrenia Bulletin, 32, 366-377, 2006. 
5. Benedetti F: The opposite effects of the opiate antagonist naloxone and the cholecystokinin antagonist proglumide on placebo analgesia. Pain, 64, 535-543, 1996.

6. Benedetti F: Placebo effects: from the neurobiological paradigm to translational implications. Neuron, 84, 623-637, 2014.

7. Bingel U, Wanigasekera V, Wiech $\mathrm{K}$, Mhuircheartaigh $\mathrm{R}$, Ploner $\mathrm{M}$, \& Tracey I: The effect of treatment expectation on drug efficacy: Imaging the analgesic benefit of the opioid remifentanil. Science Translational Medicine, 3, 70-14, 2011.

8. Botvinick M, \& Cohen J: Rubber hands "feel" touch that eyes see. Nature, 391, 756, 1998.

9. Branthwaite A, \& Cooper P: Analgesic effects of branding in treatment of headaches. British Medical Journal, 282, 1576-1578, 1981.

10. Bray H, \& Moseley G L: Disrupted working body schema of the trunk in people with back pain. British Journal of Sports Medicine, 45, 168-173, 2011.

11. Capelari E D, Uribe C, \& Brasil-Neto J P: Feeling pain in the rubber hand: integration of visual, proprioceptive, and painful stimuli. Perception, 38, 92, 2009.

12. Chae Y, Lee I S, Jung W M, Chang D S, Napadow V, Lee H, Park H J, \& Wallraven C: Decreased peripheral and central responses to acupuncture stimulation following modification of body ownership. PloS one, 9, e109489, 2014.

13. Chae Y, Lee I S, Jung W M, Park K, Park H J, \& Wallraven C: Psychophysical and neurophysiological responses to acupuncture stimulation to incorporated rubber hand. Neuroscience Letters, 591, 48$52,2015$.

14. Colloca L, \& Benedetti F: How prior experience shapes placebo analgesia. Pain, 124, 126-133, 2006.

15. Colloca L, \& Benedetti F: Placebo analgesia induced by social observational learning. Pain, 144, $28-$ 34, 2009. 
16. de Craen A J M, Tijssen J G P, Gans J, \& Kleijnen J: Placebo effect in the acute treatment of migraine: subcutaneous placebos are better than oral placebos. Journal of Neurology, 247, 183-188, 2000.

17. Ehrsson H H, Wiech K, Weiskopf N, Dolan R J, \& Passingham R E: Threatening a rubber hand that you feel is yours elicits a cortical anxiety response. Proceedings of the National Academy of Sciences, 104, 9828-9833, 2007.

18. Eippert F, Bingel U, Schoell E D, Yacubian J, Klinger R, Lorenz J, \& Büchel C: Activation of the opioidergic descending pain control system underlies placebo analgesia. Neuron, 63, 533-543, 2009.

19. Eippert F, Finsterbusch J, Bingel U, \& Büchel C: Direct evidence for spinal cord involvement in placebo analgesia. Science, 326, 404-404, 2009.

20. Haans A, IJsselsteijn W A, \& de Kort Y A: The effect of similarities in skin texture and hand shape on perceived ownership of a fake limb. Body Image, 5, 389-394, 2008.

21. Harvie D S, Broecker M, Smith R T, Meulders A, Madden V J, \& Moseley G L: Bogus visual feedback alters onset of movement-evoked pain in people with neck pain. Psychological Science, 26, 385-392, 2015.

22. Hashmi J A, Baliki M N, Huang L, Parks E L, Chanda M L, Schnitzer T, \& Apkarian A V: Lidocaine patch $(5 \%)$ is no more potent than placebo in treating chronic back pain when tested in a randomised double blind placebo controlled brain imaging study. Molecular Pain, 8, 29, 2012.

23. Hegedüs G, Darnai G, Szolcsányi T, Feldmann A, Janszky J, \& Kállai, J: The rubber hand illusion increases heat pain threshold. European Journal of Pain, 18, 1173-1181, 2014.

24. IJsselsteijn W A, de Kort Y A W, \& Haans A: Is this my hand I see before me? The rubber hand illusion in reality, virtual reality, and mixed reality. Presence: Teleoperators and Virtual Environments, 15, 455-464, 2006.

25. Lewis J S, Kersten P, McCabe C S, McPherson K M, \& Blake D R: Body perception disturbance: a contribution to pain in complex regional pain syndrome (CRPS). Pain, 133, 111-119, 2007. 
26. Lloyd D M: Spatial limits on referred touch to an alien limb may reflect boundaries of visuo-tactile peripersonal space surrounding the hand. Brain and Cognition, 64, 104-109, 2007.

27. Longo M R, Betti V, Aglioti S M, \& Haggard P: Visually induced analgesia: seeing the body reduces pain. Journal of Neuroscience, 29, 12125-12130, 2009.

28. Longo M R, lannetti G D, Mancini F, Driver J, \& Haggard P: Linking pain and the body: neural correlates of visually induced analgesia. Journal of Neuroscience, 32, 2601-2607, 2012.

29. Lopez C, Bieri C P, Preuss N, \& Mast F W: Tactile and vestibular mechanisms underlying ownership for body parts: a non-visual variant of the rubber hand illusion. Neuroscience Letters, 511, 120-124, 2012.

30. Lund K, Vase L, Petersen G L, Jensen T S, \& Finnerup N B: Randomised controlled trials may underestimate drug effects: balanced placebo trial design. Plos One, 9, e84104, 2014.

31. Mancini F, Longo M R, Kammers M P, \& Haggard P: Visual distortion of body size modulates pain perception. Psychological Science, 22, 325-330, 2011.

32. Martini M, Perez-Marcos D, \& Sanchez-Vives M V: Modulation of pain threshold by virtual body ownership. European Journal of Pain, 18, 1040-1048, 2014.

33. Mohan R, Jensen K B, Petkova V I, Dey A, Barnsley N, Ingvar M, McAuley J H, Moseley G L, \& Ehrsson H H: No pain relief with the rubber hand illusion. PLoS One, 7, e52400, 2012.

34. Montgomery G H, \& Kirsch I: Classical conditioning and the placebo effect. Pain, 72, 107-113, 1997.

35. Moseley G L: Distorted body image in complex regional pain syndrome. Neurology, $65,773-773$, 2005.

36. Moseley G L: I can't find it! Distorted body image and tactile dysfunction in patients with chronic back pain. Pain, 140, 239-243, 2008. 
37. Moseley G L, Gallace A, \& Spence C: Bodily illusions in health and disease: physiological and clinical perspectives and the concept of a cortical 'body matrix'. Neuroscience and Biobehavioral Reviews, 36, 34-46, 2012.

38. Moseley G L, Zalucki N M, \& Wiech K: Tactile discrimination, but not tactile stimulation alone, reduces chronic limb pain. Pain, 137, 600-608, 2008.

39. Nierula B, Martini M, Matamala-Gomez M, Slater M, \& Sanchez-Vives M V: Seeing an Embodied Virtual Hand is Analgesic Contingent on Colocation. The Journal of Pain, 2017.

40. Petrovic $P$, Kalso E, Petersson K M, \& Ingvar M: Placebo and opioid analgesia - imaging a shared neuronal network. Science, 295, 1737-1740, 2002.

41. Petrovic $P$, Kalso E, Petersson K M, Andersson J, Fransson P, \& Ingvar M: A prefrontal non-opioid mechanism in placebo analgesia. Pain, 150, 59-65, 2010.

42. Pollo A, Amanzio M, Arslanian A, Casadio C, Maggi G, \& Benedetti F: Response expectancies in placebo analgesia and their clinical relevance. Pain, 93, 77-84, 2001.

43. Price D D, Long S, Wilsey B, \& Rafii A: Analysis of peak magnitude and duration of analgesia produced by local anesthetics injected into sympathetic ganglia of complex regional pain syndrome patients. The Clinical Journal of Pain, 14, 216-226, 1998.

44. Price D D, Milling LS, Kirsch I, Duff A, \& Montgomery G: An analysis of factors that contribute to the magnitude of placebo analgesia in an experimental paradigm. Pain, 83, 147-156, 1998.

45. Rief W, \& Glombiewski J A: The hidden effects of blinded, placebo-controlled randomized trials: an experimental investigation. Pain, 153, 2473-2477, 2012.

46. Rief W, Nestoriuc Y, von Lilienfeld-Toal A, Dogan I, Schreiber F, Hofmann S G, Barsky A J, \& Avorn J: Differences in adverse effect reporting in placebo groups in SSRI and tricyclic antidepressant trials. Drug Safety, 32, 1041-1056, 2009. 
47. Slater M, Perez-Marcos D, Ehrsson H, \& Sánchez-Vives M V: Towards a digital body: the virtual arm illusion. Frontiers in Human Neuroscience, 2, 6, 2008.

48. Stewart-Williams S, \& Podd J: The placebo effect: dissolving the expectancy versus conditioning debate. Psychological Bulletin, 130, 324, 2004.

49. Tsakiris $M$, \& Haggard $P$ : The rubber hand illusion revisited: visuotactile integration and selfattribution. Journal of Experimental Psychology: Human Perception and Performance, 31, 80, 2005.

50. Tsakiris M: My body in the brain: a neurocognitive model of body-ownership. Neuropsychologia, 48, 703-712, 2010.

51. Vase L, Petersen G L, Riley J L, \& Price D D: Factors contributing to large analgesic effects in placebo mechanism studies conducted between 2002 and 2007. Pain, 145, 36-44, 2009.

52. Wager T D, Rilling J K, Smith E E, Sokolik A, Casey K L, Davidson R J, Kosslyn S M, Rose R M, \& Cohen J D: Placebo-induced changes in FMRI in the anticipation and experience of pain. Science, 303, 11621167, 2004. 
Figure 1. The experimental set-up, depicting the two sites on the real right arm and the artificial limb, as well as the partition obscuring view of the right arm (Figure adapted from Capelari et al. ${ }^{8}$ Permission of Pion Ltd, London).

Figure 2. The experimental procedure; three phases of thermal stimulation, split between two sites, were carried out under conditions of either synchronous or asynchronous stimulation. After baseline assessment a placebo and control treatment were applied to the rubber arm and intensity reduced at the site receiving the placebo. During post-treatment, this site was returned to baseline intensity (Figure adapted from Capelari et al. ${ }^{8}$ Permission of Pion Ltd, London).

Figure 3. Mean, standard error, and effect size (Cohen's d) for pain intensity ratings during the pre- and post-treatment phases at the placebo and control sites during both synchronous and asynchronous stimulation. Significant differences are marked with an asterisk $(* p<.025)$.

Figure 4. Mean, standard error, and effect size (Cohen's d) for pain unpleasantness ratings during the pre- and post-treatment phases at the placebo and control sites during both synchronous and asynchronous stimulation. Significant differences are marked with an asterisk $(*=p<.016)$.

Table 1. Rubber hand illusion questionnaire. 
Table 2. The procedure employed by in the experiment. Stimulation intensity was applied based on a 0100 pain rating scale.

Table 3 .Means and standard deviation for subscales of the RHI questionnaire and proprioceptive drift (cm) across conditions of synchronicity, treatment, and phase. Significance of synchronous over asynchronous stimulation is marked with an asterisk $\left({ }^{\star *}=\mathrm{p}<.01,{ }^{*}=\mathrm{p}<.05\right)$. Note, the conditioning phase was not included in analyses.

Table 4. Means and standard deviations for pain intensity across conditions of synchronicity, treatment, and phase.

Table 5. Means and standard deviations for pain unpleasantness across conditions of synchronicity, treatment, and phase.

Table 6. Correlation matrix between measures of pain intensity and unpleasantness and measures of the $\mathrm{RHI}$ at the placebo treatment site in the post-treatment phase, ${ }^{*}$ indicates a significant level of .05 , while ** indicates a significance level of .01 . 
Table 1.

RHI Questionnaire

1 It seemed as though the rubber hand were my hand.

2 It seemed as if I were feeling the pain at the location where I saw the rubber hand stimulated.

3 It seemed as though the pain I felt was caused by the painful stimulation of the rubber hand.

4 It seemed as though the touch I felt was caused by the touching of the rubber hand.

5 It seemed as if I were feeling the touch in the location where I saw the rubber hand touched.

Table 2.

\begin{tabular}{lccc}
\hline & Pre-Treatment & Conditioning & Post-Treatment \\
\cline { 2 - 4 } Placebo & 60 (2 blocks) & $30(2+1$ blocks $)$ & 60 ( 2 blocks $)$ \\
Control & 60 (2 blocks) & $60(2+1$ blocks $)$ & 60 (2 blocks) \\
\hline
\end{tabular}


Table 3.

\begin{tabular}{|c|c|c|c|}
\hline & \multicolumn{3}{|c|}{ Subscales of the RHI } \\
\hline & \multicolumn{3}{|c|}{ Ownership } \\
\hline & Pre-Treatment & Conditioning & Post-Treatment \\
\hline Placebo Synchronous & $2.30(1.81)^{* *}$ & $2.45(1.90)$ & $2.40(1.96) * *$ \\
\hline Control Synchronous & $2.45(1.79) * *$ & $2.60(1.82)$ & $2.55(1.79)^{* *}$ \\
\hline Placebo Asynchronous & $1.45(1.64)$ & $1.65(1.76)$ & $1.65(1.90)$ \\
\hline \multirow[t]{3}{*}{ Control Asynchronous } & $1.25(1.48)$ & $1.40(1.54)$ & $1.70(1.78)$ \\
\hline & \multicolumn{3}{|c|}{ Tactile Embodiment } \\
\hline & Pre-Treatment & Conditioning & Post-Treatment \\
\hline Placebo Synchronous & $2.50(1.96)$ & $2.58(1.95)$ & $2.33(1.86)$ \\
\hline Control Synchronous & $2.38(1.85)$ & $2.68(1.90)$ & $2.33(1.68)$ \\
\hline Placebo Asynchronous & $1.73(1.59)$ & $1.90(1.75)$ & $1.85(1.92)$ \\
\hline \multirow[t]{3}{*}{ Control Asynchronous } & $1.50(1.61)$ & $1.65(1.77)$ & $2.00(1.77)$ \\
\hline & \multicolumn{3}{|c|}{ Nociceptive Embodiment } \\
\hline & Pre-Treatment & Conditioning & Post-Treatment \\
\hline Placebo Synchronous & $1.68(1.84)^{* *}$ & $1.73(1.87)$ & $2.05(2.08) * *$ \\
\hline Control Synchronous & $1.63(1.75)^{* *}$ & $1.88(2.00)$ & $1.80(1.92) * *$ \\
\hline Placebo Asynchronous & $0.80(1.15)$ & $1.25(1.54)$ & $1.10(1.64)$ \\
\hline \multirow[t]{3}{*}{ Control Asynchronous } & $0.88(1.26)$ & $1.03(1.33)$ & $1.13(1.57)$ \\
\hline & \multicolumn{3}{|c|}{ Proprioceptive Drift } \\
\hline & Pre-Treatment & Conditioning & Post-Treatment \\
\hline Placebo Synchronous & $1.16(3.38)$ & $1.28(3.30)$ & $1.17(2.68)$ \\
\hline Control Synchronous & $1.37(3.44)$ & $0.03(3.25)$ & $1.93(2.17)$ \\
\hline Placebo Asynchronous & $0.89(3.23)$ & $0.71(2.62)$ & $0.22(3.10)$ \\
\hline Control Asynchronous & $1.17(3.02)$ & $0.64(4.46)$ & $-0.03(1.90)$ \\
\hline
\end{tabular}

Table 4.

\begin{tabular}{|c|c|c|c|c|}
\hline & \multicolumn{2}{|c|}{ Intensity - Synchronous Stimulation } & \multicolumn{2}{|c|}{ Intensity - Asynchronous Stimulation } \\
\hline & Placebo & Control & Placebo & Control \\
\hline Pre-treatment & $56.3(9.9)$ & $55.7(12.8)$ & $53.1(13.7)$ & $58.0(11.5)$ \\
\hline Conditioning & $29.6(11.4)$ & $57.0(12.9)$ & $27.9(13.8)$ & $59.6(15.4)$ \\
\hline Post-treatment & $50.0(14.5)$ & $60.8(14.2)$ & $54.3(20.3)$ & $58.2(20.0)$ \\
\hline Pre-Post Difference & $-6.3(14.5)$ & $5.1(10.8)$ & 1.2 (13.9) & $0.2(15.1)$ \\
\hline
\end{tabular}


Table 5.

\begin{tabular}{lcccc}
\hline & \multicolumn{2}{c}{$\begin{array}{c}\text { Unpleasantness - Synchronous } \\
\text { Stimulation }\end{array}$} & \multicolumn{2}{c}{$\begin{array}{c}\text { Unpleasantness - Asynchronous } \\
\text { Stimulation }\end{array}$} \\
\cline { 2 - 5 } & \multicolumn{2}{c}{ Placebo } & Control & \multicolumn{2}{c}{ Placebo } & Control \\
\cline { 2 - 5 } Pre-treatment & $46.4(13.4)$ & $46.6(13.9)$ & $43.4(17.9)$ & $51.3(17.1)$ \\
Conditioning & $21.0(10.3)$ & $48.7(14.4)$ & $21.1(16.5)$ & $53.2(20.7)$ \\
Post-treatment & $43.5(16.3)$ & $53.8(19.0)$ & $46.9(23.6)$ & $52.0(22.6)$ \\
Pre-Post Difference & $-2.9(15.2)$ & $7.2(10.7)$ & $3.5(13.4)$ & $0.7(13.5)$ \\
\hline
\end{tabular}


Table 6.

\begin{tabular}{|c|c|c|c|c|c|c|}
\hline & Intensity & Intensity Pre - Post & $\begin{array}{c}\text { Intensity Placebo - } \\
\text { Control }\end{array}$ & Unpleasantness & $\begin{array}{c}\text { Unpleasantness Pre } \\
\text { - Post }\end{array}$ & $\begin{array}{c}\text { Unpleasantness } \\
\text { Placebo-Control }\end{array}$ \\
\hline $\begin{array}{l}\text { Ownership Pre } \\
\text { Tactile Embodiment }\end{array}$ & -.053 & -.249 & .111 & -.052 & -.171 & .107 \\
\hline Pre & -.005 & -.280 & .290 & -.006 & -.209 & .284 \\
\hline Pain Embodiment Pre & -.411 & -.433 & .247 & -.332 & $-.467 *$ & .199 \\
\hline Post & -.121 & -.384 & .396 & -.071 & -.125 & .347 \\
\hline Pain Embodiment Post & -.341 & $-.483 *$ & .287 & -.205 & -.335 & .246 \\
\hline
\end{tabular}


Figure 1.

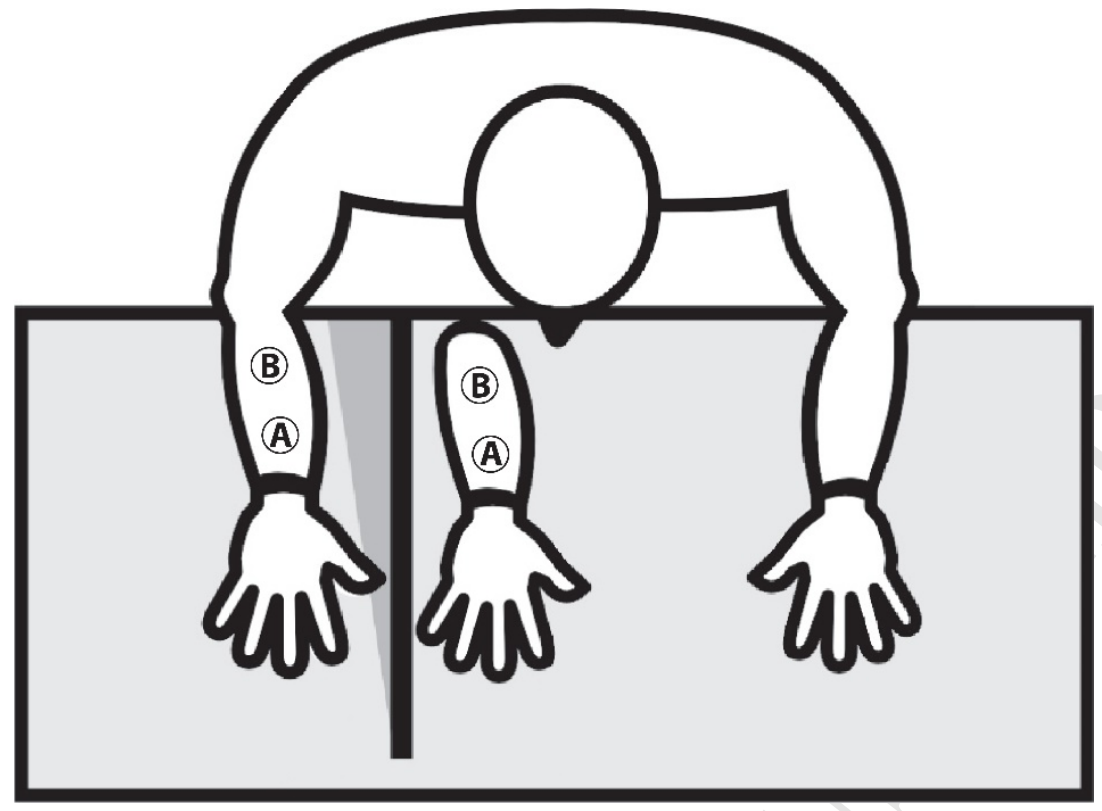

Figure 2.
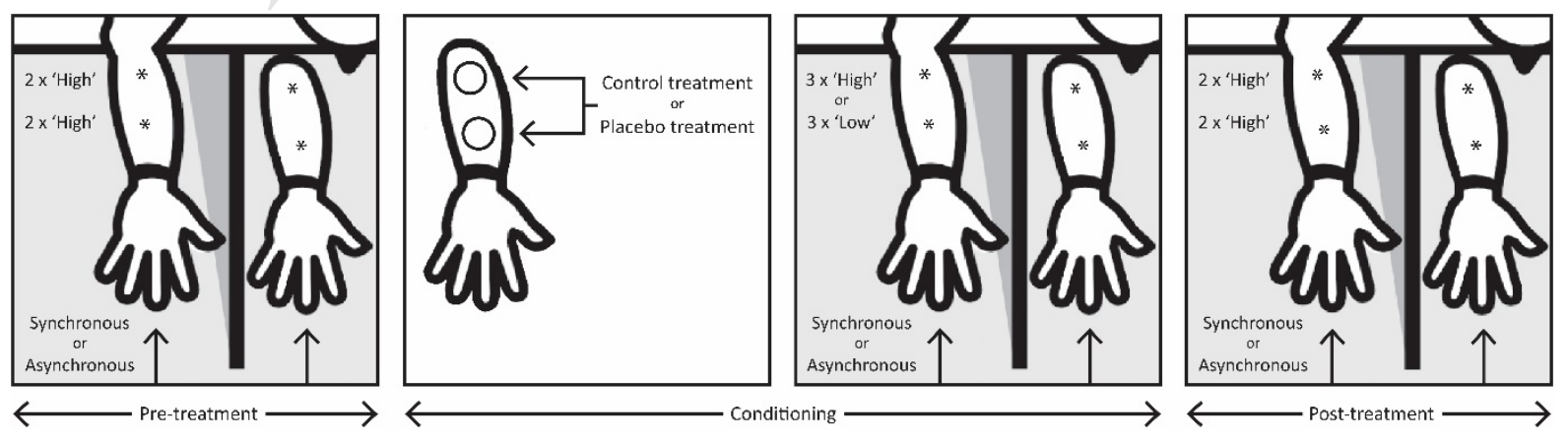
Figure 3.
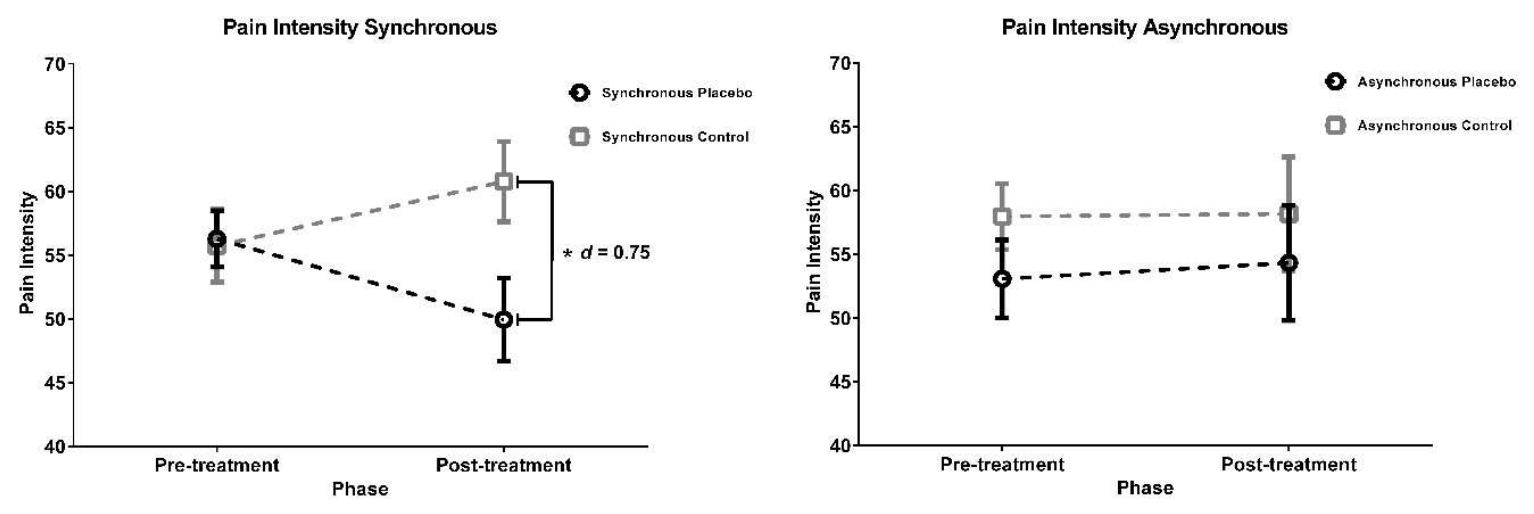

Figure 4.
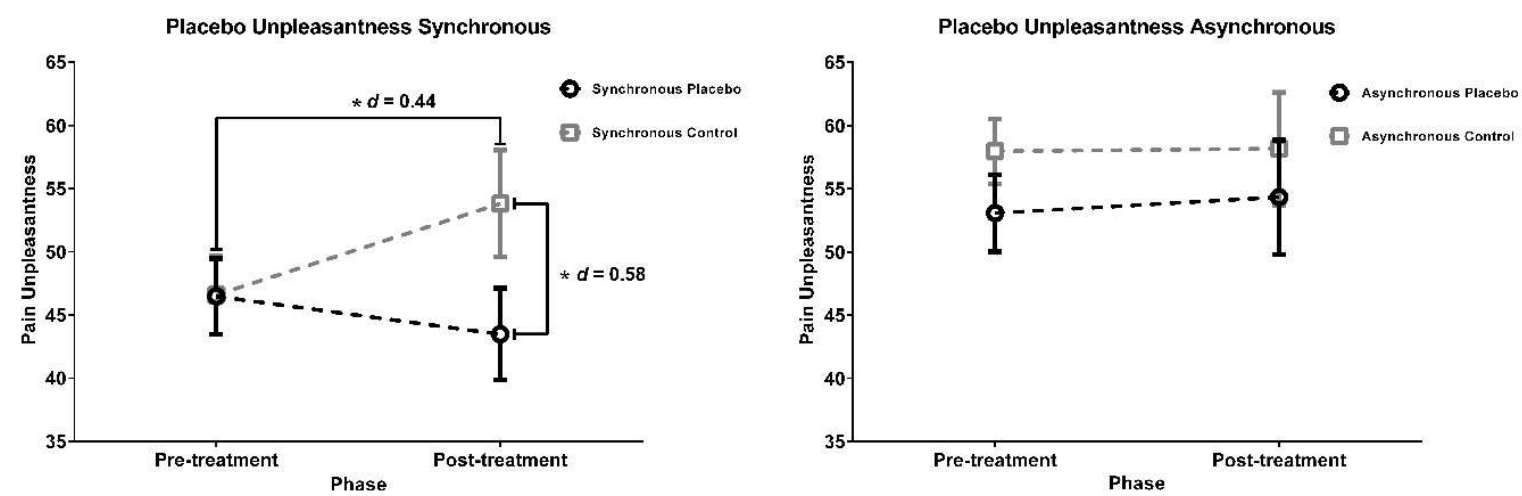


\section{Placebo Analgesia from a Rubber Hand - Highlights}

- The rubber hand illusion is an illusion of embodiment over an artificial limb.

- A sham analgesic applied to the rubber hand can produce placebo analgesia.

- Embodiment may influence expectations of treatment efficacy and the placebo effect. 